\title{
VHDL Performance Analysis of Correlation Radar with Distance, Speed and Angle Measurement for Automotive Application
}

\author{
Pankaj Soni \\ M. Tech. Scholar \\ NRI Institute of Information Science \& Technology. \\ Bhopal
}

\begin{abstract}
Radars are general devices for defense, survey \& vehicle guidance systems. However the sophisticated design \& requirements of costly devices, makes it out of reach to the daily applications. Correlation radars are used as anti collision system for auto - motive applications. Objective of this system is to design simple, cost effective architecture of the correlation radar. As a consequence, this system will reduce accidents and enhance road safety.

This paper proposed an approach to make the device simple, cheap \& much compatible for the automobiles. As a result, it can be used for automated vehicle derive system as well as collision avoidance system. We are presenting a VHDL design for the correlation radar, which is capable to measure distance, relative speed and angle. The proposed radar uses the two receiver concept to measure the angle of the object. The capability to angle measurement will guide about direction of obstacle and also increases the application area of this correlation radar.

Principle of this system is to avoid collision between the equipped vehicle and the one in front, or other kind of obstacles (pedestrians, animals, etc). As a result this radar can replace the existing previous version of radar and improve the road safety.
\end{abstract}

\section{Keywords}

Correlation radar, automotive radars, anti - collision system, VHDL design, pseudo random sequence, XC 9500 CPLDs.

\section{INTRODUCTION}

Automotive radar devices are now appearing on many transport and luxury passenger motor vehicles. Typical automotive radar is mounted behind the front grille of a vehicle at a height of less than 1 meter, where it is able to interrogate the road ahead and the adjacent traffic lanes forward of the vehicle's location. Anti-collision systems are part of these innovative electronic devices used to build intelligent vehicles with a maximum safety.

Principle of the system is to avoid collision between the equipped vehicle and the one in front \& other kind of obstacles. The safety distance is the minimum distance required to stop a vehicle. Drivers need assistance to perform an efficient estimation of distances on the road under bad vision conditions (rain, fog, snow etc). The radar is not limited by weather conditions. Our system can determine the distance between the vehicle and the obstacle. By periodically computing this distance, the system can also estimate the relative speed and angle between the equipped vehicle and the obstacles.

\author{
Puran Gour \\ Professor \\ NRI Institute of Information Science \& Technology. \\ Bhopal
}

\section{LITERATURE SURVEY}

The concept of automotive radar is not new. It was proposed from the invention of radar, but because of unavailability of technology, it was never taken seriously. Past two decades technology made it feasible. So many people proposed their concepts for making better automotive radar. Some of the relevant papers are discussed here:-

Firstly, Lounis DOUADI, Pascal DELOOF, Yassin ELHILLALI described the principle of distance measurement with correlation radar and its contribution on real time implementation. Two tests were performed to check the good functioning of the VHDL code generator and the correlator itself. In the second test a distance measurement was performed using a cable. The results confirmed the good functioning of the correlator and the correlation generator [1]. Shruti Bhargava, Snehprabha Lad proposed the implementation of a FPGA based design, which is capable to measure the distance of other vehicle as glowing speed \& it can also differentiate the signals from other large number of radars transmitting at same occurrence in same road, which made this radar useful for large traffic area. The paper describes the correlation code matching algorithm \& it implementation on FPGA using VHDL [2].

Yahya S. H. Khraisat proposed system to reduce traffic accidents and danger that faces the driver and the vehicles as a result of the sudden collision. The proposed system covers distance up to $30 \mathrm{~m}$. However, if the distance between vehicle $\&$ object be less than $2 \mathrm{~m}$, the system will produce sound like alarm, to alert the driver about danger and the driver can take the appropriate decision to avoid collision [3].

S. Le Beux, V. Gagné, E.M. Aboulhamid and Ph. Marquet proposed anti-collision radar, which help to prevent accidents by detecting obstacles in front of vehicles. This task was realiezed on a correlator, which searches for similarities between an emitted and a received wave. Then other modules can use the information, produced by the correlator to compute the distance and the relative speed between the moving vehicle and the obstacle [4].

Hermann Rohling, Marc-Michael Meinecke proposed new waveform design, which has all advantages of FMCW radars. This system needs an extremely low measurement time (10ms) in a radar sensor [5].

\section{PROPOSED WORK}

\subsection{Correlation radar}

To measure the angle of object with measurement of distance and speed, So that the correlation radar becomes more effective to be used in auto - motive applications.

Digital correlation radar is shown in Fig 1. The correlation is the sum of sample products $x$ and $y$, with $y$ being only shifted in time. 


$$
C_{x y}(k)=\Sigma^{N}{ }_{i=0} x(i) y(k+i)
$$

Where $C_{\mathrm{xy}}$ is the correlation, $N$ is the number of samples and $k$ is the time shift.

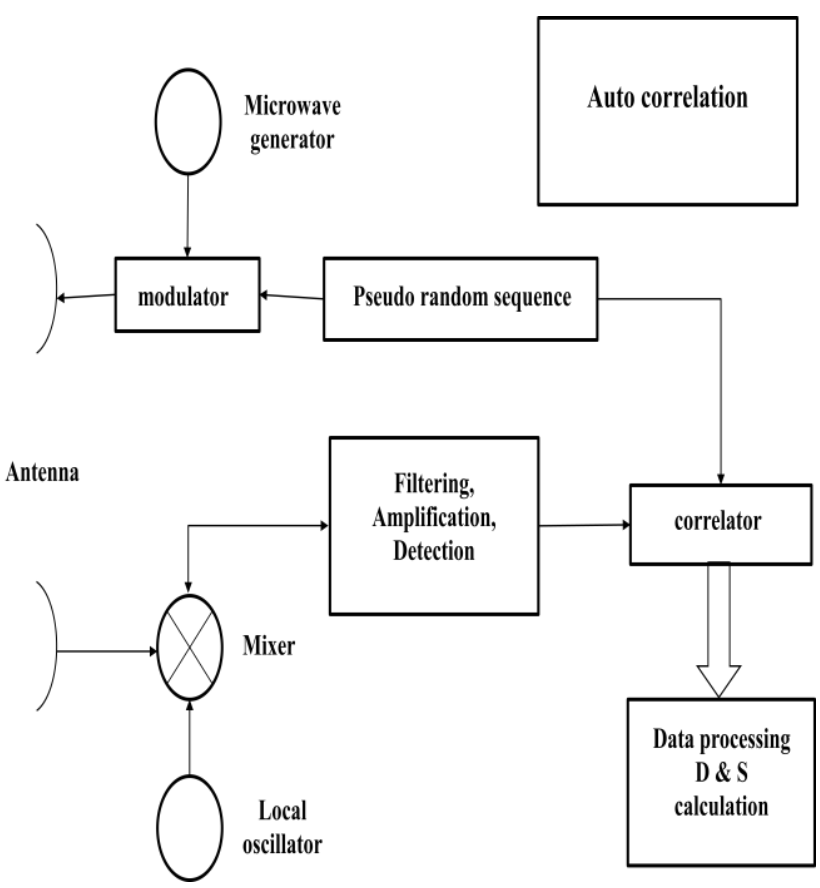

Fig 1: Block diagram of the correlation radar

\subsection{Block diagram}

The block diagram of the proposed model is shown in fig 2 . The system uses the two receiver concept for measuring the angle.

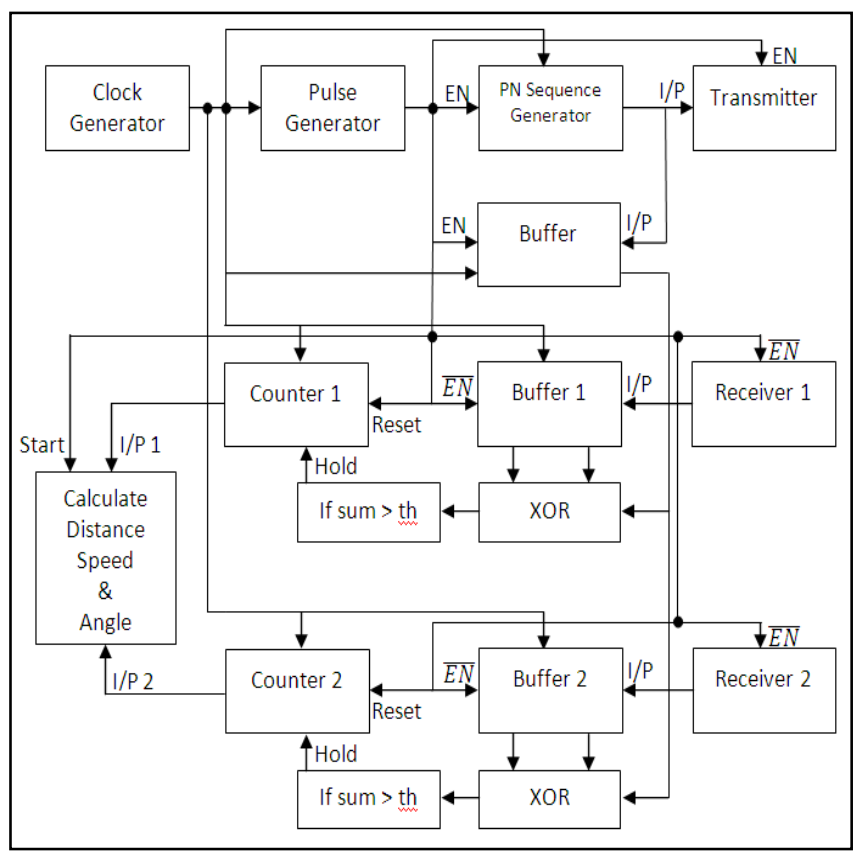

Fig 2: Block diagram of the proposed model

The working of the complete system can be easily understood by the block diagram -
1. Clock Generator: this block is used for generating the clock for all blocks of system.

2. Pulse Generator: this section generates a high pulse of duration 128 clock and then remains low for 1000 clock. This pulse synchronizes the blocks and avoids the crosstalk between blocks.

3. PN-Sequence Generator: this block generates a PN sequence by shift register. The length of shift register in present system is eight. The PN sequence generated by this block is transmitted for obstacle detection.

4. Buffer: the work of buffer is to store the transmitted or received (Buffer 1 and buffer 2) signal for performing the correlation between transmitted and received signals.

5. Counter: This block is used to measure the delay between transmitted and received signal in terms of clock counts.

6. XOR: this is used for calculating the correlation (similarity) between TX buffer and RX buffer. The number of ones in the output of this block shows the similar bits.

7. Threshold Detector: it compares the number of one's at the output of the XOR with threshold value to detect the reflected signal. For this system, we are taking value of threshold 100

8. Calculate Distance, Speed and Angle: this block estimates the distance, speed and angle from the values count 1 and count2.

\section{RESULTS \& DISCUSSIONS}

Fig. 3 shows the RTL schematic view of previous work, implemented on Xilinx 8.1 ISE, done by Lounis DOUADI, Pascal DELOOF, Yassin ELHILLALI [1]. In this work, correlation radar is used to measure distance and speed between equipped vehicle and obstacles.

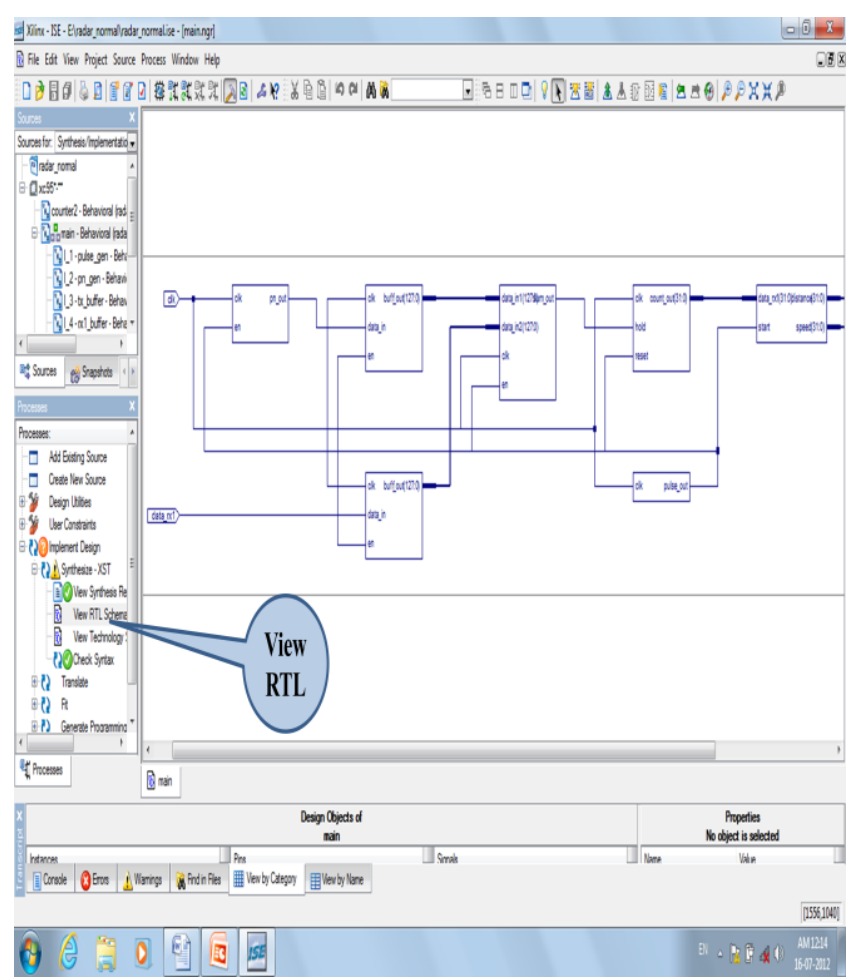

Fig 3: RTL model of previous work, implemented in Xilinx 8.1 ISE.

Fig. 4 shows the RTL schematic view of proposed work, implemented on Xilinx 8.1 ISE. In this work, correlation radar 
is used to measure angle with distance and speed measurement between equipped vehicle and obstacles.

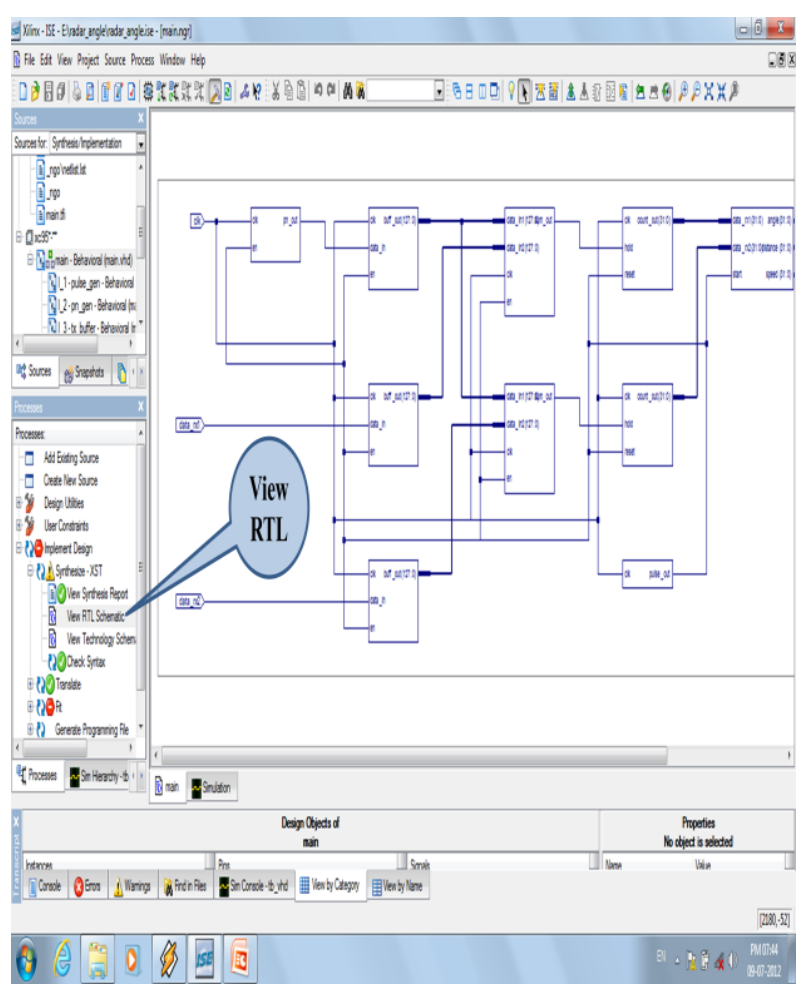

Fig 4: RTL model of proposed work, implemented in Xilinx 8.1 ISE.

Fig. 5 shows the HDL synthesis report of previous work, which describes the Macro Statistics of synthesis [1].

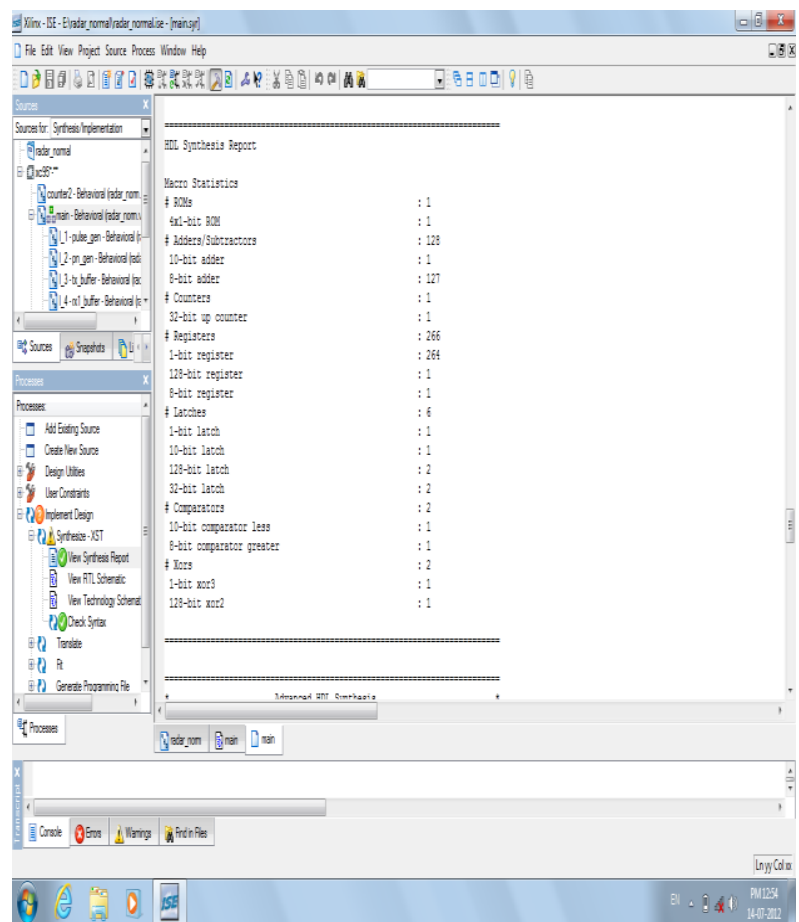

Fig 5: HDL synthesis report of previous work.

Fig. 6 shows similar the HDL synthesis report of proposed work.

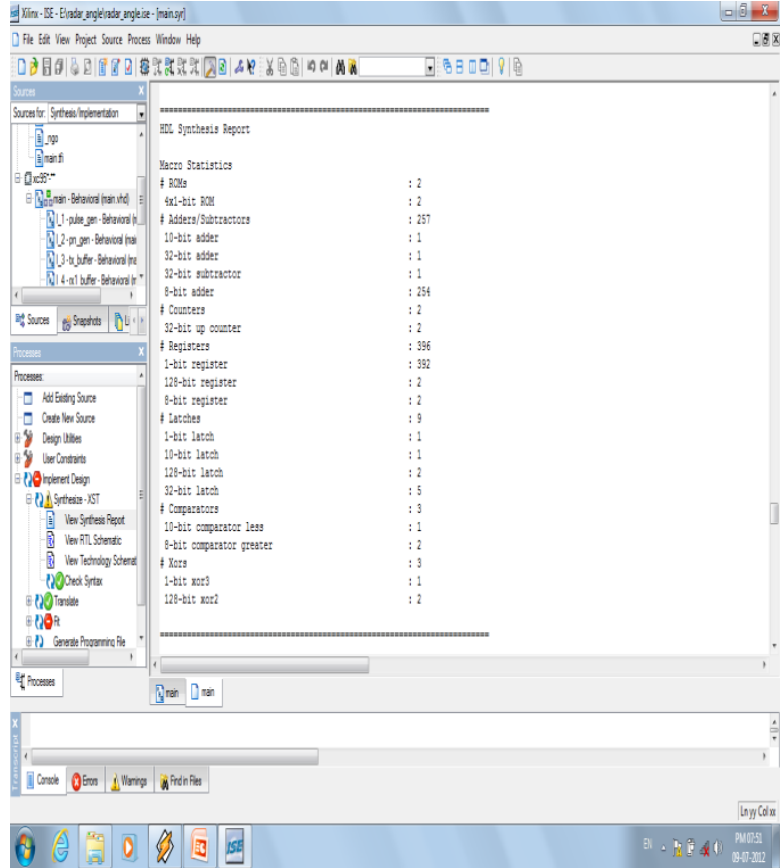

Fig 6: HDL synthesis report of proposed work.

After simulation of the proposed and previous work in VHDL, we are analyzing the proposed simulation results with previous simulation results, tabulated below:-

Table 1. Analysis of proposed simulation with previous simulation

\begin{tabular}{|c|c|c|c|}
\hline $\begin{array}{c}\text { S. } \\
\text { No. }\end{array}$ & Components & $\begin{array}{c}\text { Utilization } \\
\text { in proposed } \\
\text { work }\end{array}$ & $\begin{array}{c}\text { Utilization in } \\
\text { previous } \\
\text { work }\end{array}$ \\
\hline 1. & $\begin{array}{c}\text { Adders/ Sub } \\
\text { tractors }\end{array}$ & 257 & 128 \\
\hline 2. & 32 bit up- counter & 2 & 1 \\
\hline 3. & Registers & 396 & 266 \\
\hline 4. & Latches & 9 & 6 \\
\hline 5. & I/Os & 100 & 67 \\
\hline 6. & Bels & 14208 & 7685 \\
\hline 7. & Flip- flops & 1155 & 763 \\
\hline 8. & Input buffers & 3 & 64 \\
\hline 9. & Output buffers & 96 & 16 second \\
\hline 10. & Elapsed time & 26 second & \\
\hline
\end{tabular}

\section{CONCLUSION}

After the analysis of proposed work \& previous work from the table, we can conclude that if we will measure angle with measurement of distance \& speed, the device XC 9500 CPLD will do simulation within 26 second. According to simulation results, it has been shown that the proposed algorithm is an 
efficient solution for well-known correlation radar with angle measurement capability. In future, we can do same simulation in $3 \mathrm{D}$ space. So that height and shape of obstacles can also be measured with distance, speed and angle measurement.

\section{REFERENCES}

[1] Lounis DOUADI, Pascal DELOOF, Yassin ELHILLALI, "Real time implementation of reconfigurable correlation radar for road anti-collision system”, 24 April 2008, IEEE international Conference.

[2] Shruti Bhargava, Snehprabha Lad, "Implementation of automotive radar based on correlation radar", Dec 2011, IJCTEE.

\section{AUTHOR'S PROFILE}

Mr. Pankaj Soni has received his B.E. Degree in Electronics \& Communication on June 2008 from Rajiv Gandhi Proudyogiki Vishwavidyalaya (RGPV), Bhopal, India and currently pursuing $M$. Tech. Degree in Micro Electronics \& VLSI Design from Rajiv Gandhi Proudyogiki Vishwavidyalaya (RGPV), Bhopal, India.

Prof. Puran Gour has received his B.E. Degree from Amravati University, Amravati, India and M. Tech. Degree
[3] Yahya S. H. Khraisat, "Simulation of the 24 GHz Short Range Automotive Radar", 2011, European Journal of Scientific Research, Vol.67, No.1, pp. 75-82.

[4] S. Le Beux, V. Gagné, E.M. Aboulhamid, Ph. Marquet and J.-L. Dekeyser, "Hardware/Software Exploration for an Anti-collision Radar System", 09 Aug 2006, IEEE international Conference.

[5] Hermann Rohling, Marc - Michael Meinecke, "Waveform Design Principles for Automotive Radar Systems", 2001, IEEE international Conference on China, pp. 1-4.

[6] Douglas L. Perry, VHDL Programming by Examples, Tata McGraw-Hill Publishing Company Limited.

from MANIT, Bhopal, India. He is currently working as an Professor in department of Electronics \& Communication, NRI Institute of Information Science and Technology, Bhopal, India. He has over ten years teaching experience. His research interest is in Wireless Communication, Microwave Communication and VLSI Design. 